\title{
CLASSIFICATION OF COHEN-MACAULAY MODULES OF COVARIANTS FOR SYSTEMS OF BINARY FORMS
}

\author{
BRAM BROER
}

(Communicated by Eric Friedlander)

\begin{abstract}
For every module of covariants for a system of binary forms a formula is given, measuring to what extent Stanley's functional equation fails to be satisfied. As an application a new proof is given for the classification of the Cohen-Macaulay modules of covariants for systems of binary forms.
\end{abstract}

\section{INTRODUCTION}

Let $V$ and $M$ be two finite-dimensional $\mathrm{SL}_{2}$-modules. The vector space of polynomial maps $V \rightarrow M$ commuting with the $\mathrm{SL}_{2}$-actions is a finitely generated graded module $B(M)$ over the algebra $B$ of invariant polynomial functions on $V$. It is called the module of covariants for $V$ of type $M$. The ring of invariants is finitely generated and Hochster and Roberts [7] showed that it is Cohen-Macaulay, in fact, even Gorenstein.

If the highest weights of $M$ are smaller than $s-2$, where $s$ is an easily computed integer depending on $V$, Stanley [10] proved in 1979 that the generating function of $B(M)$ satisfies a functional equation of the kind

$$
\mathscr{G}(B(M) ; t)=(-1)^{d} t^{-\operatorname{dim} V \mathscr{G}\left(B(M), t^{-1}\right)}
$$

and made some conjectures.

Early in 1989 Van den Bergh proved these conjectures for $\mathrm{SL}_{2}$ (and later for general reductive groups, see [2]), by showing that these modules of covariants of small type are Cohen-Macaulay graded $B$-modules. In the spring of the same year we described [3] a new method of calculating generating functions and found some examples of modules of covariants not satisfying the functional equation together with a formula for the deviation from it. Furthermore, we classified the Cohen-Macaulay modules of covariants for certain classes of representations $V$. Subsequently, in the fall Van den Bergh [1] finished the classification for all $V$.

In this article we complete the picture by calculating the deviation from Stanley's functional equation for all modules of covariants and giving it an interpretation. Satisfying the functional equation turns out to be equivalent to being

Received by the editors April 17, 1992.

1991 Mathematics Subject Classification. Primary 13C14; Secondary 14L30.

(C) 1993 American Mathematical Society $0002-9939 / 93 \$ 1.00+\$ .25$ per page 
Cohen-Macaulay. We reprove the complete classification in a more elementary way.

The first sections are concerned with generating functions and complete the results in [3]. Next we show that there are at most two nonvanishing local cohomology groups. Finally we prove the classification of Cohen-Macaulay modules of covariants in full and give an interpretation of the deviation from Stanley's functional equation.

\section{Notation}

Let $\mathbf{k}$ be an algebraically closed field of characteristic zero. Let $G$ := $\operatorname{SL}(2, \mathbf{k})$ and $R_{p}$ be the irreducible representation on the binary forms of degree $p$. The subgroup of diagonal matrices is denoted by $H$. We fix a representation $V=R_{d_{1}} \oplus \cdots \oplus R_{d_{m}}$, where we suppose that $d_{i}$ is even if and only if $i \leq e$, and $e=\operatorname{dim} V^{H}$.

Write $A:=\mathbf{k}[V]$ for the algebra of functions on $V, B:=A^{G}$ for the ring of invariants, and $B(M):=(A \otimes M)^{G}$ for the module of covariants of type $M$, where $M$ is any $G$-module. They all have a natural $\mathbb{N}^{m}$-graded structure. For any $\mathbb{Z}^{m}$-graded vector space $W=\bigoplus_{\mathbf{i} \in \mathbb{Z}^{m}} W_{\mathbf{i}}$ we define the generating function

$$
\mathscr{G}(W)=\mathscr{G}(W ; \mathbf{t}):=\sum_{\mathbf{i} \in \mathbb{Z}^{m}} \operatorname{dim} W_{\mathbf{i}} \mathbf{t}^{\mathbf{i}},
$$

where $\mathbf{t}^{\mathbf{i}}:=\prod_{n=1}^{m} t_{n}^{i_{n}}$. By $\mathbf{t}^{-1}$ we mean $\left(t_{1}^{-1}, \ldots, t_{m}^{-1}\right)$.

Using the notation $\lceil z\rceil$ for the smallest integer greater than or equal to $z$, we define

$$
f^{(n)}(t):=\prod_{i=0}^{\lceil n / 2\rceil-1}\left(1-x^{n-2 i} t\right),
$$

and $f(x, \mathbf{t}):=\prod_{k=1}^{m} f^{\left(d_{k}\right)}\left(x, t_{k}\right)$. Now write $s$ for the degree of $f$ with respect of $x$, and define $\beta_{i}, \phi_{i}$, and $\alpha_{i}^{p}$ by

$$
f(x, \mathbf{t})=\sum_{i=0}^{s} \beta_{i}(\mathbf{t}) x^{i}, \quad \frac{1}{f(x, \mathbf{t})}=\sum_{i=0}^{\infty} \phi_{i}(\mathbf{t}) x^{i}, \quad \alpha_{i}^{p}=\beta_{p-i}-\beta_{i+p+2} .
$$

Finally $E:=\prod_{k=1}^{e}\left(1-t_{k}\right), T:=\prod_{i=1}^{m}\left(-t_{i}\right)^{\left\lceil d_{i} / 2\right\rceil}$, and $\tau:=\sum_{i-1}^{m}\left\lceil d_{i} / 2\right\rceil$. Then $\operatorname{dim} V=2 \tau+e$.

Lemma 1.1. Let $p \in \mathbb{Z}$.

(i) We have

$$
\sum_{i} \alpha_{i}^{p} \mathscr{G}\left(B\left(R_{i}\right), \mathbf{t}\right)=\frac{\phi_{-p}-\phi_{-p-2}}{E} .
$$

(ii) $\alpha_{i}^{s-2+p}(\mathbf{t})=-T \alpha_{i}^{-p}\left(\mathbf{t}^{-1}\right)$.

(iii) $\beta_{0}=\phi_{0}=1$ and $\sum_{i=0}^{k} \phi_{i} \beta_{k-i}=0$ for $k \geq 1$.

Proof. The first statement can be proved as [3, Proposition 3.1], the second statement is just [3, Lemma 4.2.3], and (iii) follows from $f \cdot \frac{1}{f}=1$ and the Cauchy product of power series. 


\section{Functional equations}

We write

$$
\tilde{\mathscr{G}}_{p}=\tilde{\mathscr{G}}_{p}(\mathbf{t}):=\mathscr{G}\left(B\left(R_{p}\right) ; \mathbf{t}\right)+(-1)^{\operatorname{dim} V}\left(t_{1}^{d_{1}+1} \cdots t_{m}^{d_{m}+1}\right)^{-1} \mathscr{G}\left(B\left(R_{p}\right) ; \mathbf{t}^{-1}\right)
$$

for the deviation from Stanley's functional equation. We have the following basic result.

Proposition 2.1. (i) For $0 \leq p<s-2$ we have $\widetilde{\mathscr{G}}_{p}=0$.

(ii) For $k \geq 0$ we have $\widetilde{\mathscr{G}}_{s-2+k}=-\psi_{k} /(E T)$, where $\psi_{k}(\mathbf{t})$ is the coefficient of $x^{k}$ in the Maclaurin expansion with respect to $x$ of

$$
\frac{\left(1-x^{2}\right)}{f(x, \mathbf{t}) f\left(x, \mathbf{t}^{-1}\right)}
$$

Proof. The first statement is proved in [3, Proposition 6.4] and is well known. Using this fact and $\sum_{i \geq 0} \alpha_{i}^{s-2+k}(\mathbf{t}) \mathscr{G}\left(B\left(R_{i}\right) ; \mathbf{t}\right)=0$ for $k \geq 0$, by Lemma 1.1(i), we have

$$
\begin{aligned}
\sum_{i \geq s-2} \alpha_{i}^{s-2+k}(\mathbf{t}) \tilde{\mathscr{G}}_{i} & =\sum_{i \geq 0} \alpha_{i}^{s-2+k}(\mathbf{t}) \tilde{\mathscr{G}}_{i} \\
& =(-1)^{\operatorname{dim} V}\left(t_{1}^{d_{1}+1} \cdots t_{m}^{d_{m}+1}\right)^{-1} \sum_{i \geq 0} \alpha_{i}^{s-2+k}(\mathbf{t}) \mathscr{G}\left(B\left(R_{i}\right) ; \mathbf{t}^{-1}\right) \\
& =(-1)^{\operatorname{dim} V}\left(t_{1}^{d_{1}+1} \cdots t_{m}^{d_{m}+1}\right)^{-1} \sum_{i \geq 0}-T \alpha_{i}^{-k}\left(\mathbf{t}^{-1}\right) \mathscr{G}\left(B\left(R_{i}\right) ; \mathbf{t}^{-1}\right) \\
& =-T(-1)^{\operatorname{dim} V}\left(t_{1}^{d_{1}+1} \cdots t_{m}^{d_{m}+1}\right)^{-1} \frac{\left(\phi_{k}\left(\mathbf{t}^{-1}\right)-\phi_{k-2}\left(\mathbf{t}^{-1}\right)\right)}{E\left(\mathbf{t}^{-1}\right)} \\
& =\frac{-1}{E T}\left(\phi_{k}\left(\mathbf{t}^{-1}\right)-\phi_{k-2}\left(\mathbf{t}^{-1}\right)\right) .
\end{aligned}
$$

Using $\alpha_{s-2+j}^{s-2+k}=\beta_{k-j}$, for $j \geq 0$, we established for all $k \geq 0$

$$
\sum_{j \geq 0} \beta_{k-j}(\mathbf{t}) \tilde{\mathscr{G}}_{s-2+j}=\frac{-1}{E T}\left(\phi_{k}\left(\mathbf{t}^{-1}\right)-\phi_{k-2}\left(\mathbf{t}^{-1}\right)\right)
$$

So

$$
\sum_{i=0}^{k} \sum_{j \geq 0} \phi_{i} \beta_{(k-i)-j}(\mathbf{t}) \widetilde{\mathscr{G}}_{s-2+j}=\frac{-1}{E T} \sum_{i=0}^{k} \phi_{i}(\mathbf{t})\left(\phi_{k-i}\left(\mathbf{t}^{-1}\right)-\phi_{k-i-2}\left(\mathbf{t}^{-1}\right)\right),
$$

and using Lemma 1.1(iii) we get

$$
\tilde{\mathscr{G}}_{s-2+k}=\frac{-1}{E T} \sum_{i=0}^{k} \phi_{i}(\mathbf{t})\left(\phi_{k-i}\left(\mathbf{t}^{-1}\right)-\phi_{k-i-2}\left(\mathbf{t}^{-1}\right)\right)=\frac{-\psi_{k}}{E T} .
$$

This completes the proof.

\section{DegREES OF GENERATING FUNCTIONS}

For a polynomial $p \in \mathbb{Z}\left[t_{1}, \ldots, t_{m}\right]$ we write $\operatorname{deg}_{i} p$ for the degree of $p$ with respect to $t_{i}$ and $\operatorname{deg} p$ for the total degree. For a rational function $p / q$ 
we define as usual $\operatorname{deg}_{i}(p / q)=\operatorname{deg}_{i} p-\operatorname{deg}_{i} q$ and $\operatorname{deg}(p / q)=\operatorname{deg} p-\operatorname{deg} q$. In this section we will calculate the degree of $\mathscr{G}\left(B\left(R_{i}\right), \mathbf{t}\right)$ if $i \geq s-2$.

We start with a lemma.

Lemma 3.1. (i) If $e<m$ then $\operatorname{deg} \phi_{k}=k$; if $e=m$ then $\phi_{2 k+1}=0$ and $\operatorname{deg} \phi_{2 k}=k$.

(ii) If $e<j \leq m$ then $\operatorname{deg}_{j} \phi_{k}=k$ and if $1 \leq j \leq e$ then $\operatorname{deg}_{j} \phi_{2 k}=k$ and $\operatorname{deg}_{j} \phi_{2 k+1}=k$ provided that $e \neq m$.

Proof. The Maclaurin expansion of $1 /\left(1-s x^{n}\right)$ is $1+s x^{n}+s^{2} x^{2 n}+s^{3} x^{3 n}$ $+\cdots$. Now $\frac{1}{f}$ is a product of such terms with various $n$ and various $t_{i}$ for $S$.

Suppose $e<j \leq m$. Then $f$ contains a unique factor $1-x t_{j}$. So $\phi_{k}$ contains $t_{j}^{k}$ as the highest $j$-degree term. So $\operatorname{deg}_{j} \phi_{k}=k$.

If $1 \leq j \leq e$, then $f$ contains the factor $1-x^{2} t_{j}$. So in $\phi_{2 k}$ the term of highest $j$-degree is $t_{j}^{k}$ and $\operatorname{deg}_{j} \phi_{2 k}=k$. If $e<m$ then $t_{j}^{k}\left(t_{e+1}+\cdots+t_{m}\right)$ is part of the highest $j$-degree, and $\operatorname{deg}_{j}\left(\phi_{2 k+1}\right)=k$. If $e=m$, then clearly $\phi_{2 k+1}=0$. This proves (ii); the first statement is proved analogously.

The following proposition extends [3, Proposition 7.5.1], saying, for example, that the total degree of $\mathscr{G}\left(B\left(R_{p}\right), \mathbf{t}\right)$ is less than or equal to $-\operatorname{dim} V$ if $p<$ $s-2$. This follows easily from Lemma 2.1(i). Recall $\tau:=\sum_{i-1}^{m}\left\lceil d_{i} / 2\right\rceil$.

Proposition 3.1. Let $k \geq 0$.

(i) If $e<m$, then

$$
\operatorname{deg} \mathscr{G}\left(B\left(R_{s-2+k}\right), \mathbf{t}\right)=-\operatorname{dim} V+\tau+k .
$$

(ii) If $e=m$, then $B\left(R_{2 k+1}\right)=0$ and $\operatorname{deg} \mathscr{G}\left(B\left(R_{s-2+2 k}\right)\right.$, t) $=-\operatorname{dim} V+$ $\tau+k$.

(iii) If $e<i \leq m$, then $\operatorname{deg}_{i} \mathscr{G}\left(B\left(R_{s-2+k}\right)\right.$, t $)=k-\left\lceil d_{i} / 2\right\rceil$.

(iv) If $1 \leq i \leq e$, then $\operatorname{deg}_{i} \mathscr{G}\left(B\left(R_{s-2+2 k}\right), \mathbf{t}\right)=\operatorname{deg}_{i} \mathscr{G}\left(B\left(R_{s-2+2 k+1}\right), \mathbf{t}\right)=$ $k-d_{1} / 2$.

Proof. The lowest degree of a term in the denominator minus the lowest degree of a term in the numerator of $\mathscr{G}\left(B\left(R_{s-2+k}\right) ; \mathbf{t}^{-1}\right)$ is just the degree $\delta$ of $\mathscr{G}\left(B\left(R_{s-2+k}\right) ; \mathrm{t}\right)$. Using Proposition 2.1 (ii) one sees that $-\operatorname{dim} V-\delta$ equals the lowest degree of a term in $\psi_{k} / T$, which is just $-\tau-k$ if $e<m$, and $-\tau-k / 2$ if $e=m$ and $k$ is even.

So $\delta=-\operatorname{dim} V+\tau+k$ if $e<m$ and $\delta=-\operatorname{dim} V+\tau+k / 2$ if $e=m$ and $k$ is even. This proves (i) and (ii). The remaining statements are proved analogously.

\section{Depth estimates}

From now on we only consider the total grading and write $\mathscr{G}\left(B\left(R_{i}\right)\right)=$ $\mathscr{G}\left(B\left(R_{i}\right), t\right)$ for the corresponding single-variable Hilbert series. Let $R$ be any finitely generated $d$-dimensional $\mathbb{N}$-graded $\mathbf{k}$-algebra, with $R_{0}=\mathbf{k}$. Write $R^{+}$for the unique maximal graded ideal and $\delta$ for the degree of $\mathscr{G}(R)$ as a rational function. Write $H_{R^{+}}^{i}(N)$ for the $i$ th local cohomology group of the graded module $N$ in the category of graded $R$-modules. See [6] for some of its properties; the most important being that $\operatorname{dim} N$ (resp. $\operatorname{depth}_{R^{+}}(N)$, i.e., 
the length of the longest regular sequence on $N$ consisting of homogeneous elements of $R^{+}$) is the largest (resp. smallest) index $i$ such that $H_{R^{+}}^{i}(N) \neq 0$. For any graded $R$-module $N$ the graded dual is defined as

$$
N^{\vee}:=\bigoplus_{n \in \mathbb{Z}} \operatorname{Hom}_{\mathbf{k}}\left(N_{-n}, \mathbf{k}\right) ;
$$

it is a graded $R$-module.

Lemma 4.1. Suppose $R$ is Gorenstein. For any finitely generated graded $R$ module $N$

$$
H_{R^{+}}^{i}(N)^{\vee} \simeq \operatorname{Ext}_{R}^{d-i}(N, R[\delta]),
$$

where $R[\delta]_{i}:=R_{\delta+i}$.

Proof. See [6, Proposition 2.1.6].

Using this graded duality, we prove that at most two local cohomology groups of modules of covariants do not vanish. We start with a lemma concerning the support. Write $\pi: V \rightarrow V / / G$ for the quotient morphism.

Lemma 4.2. The support of $\operatorname{Ext}_{B}^{i}(A, B)$ is contained in $\pi\left(V^{H}\right)$.

Proof. Let $x \notin \pi\left(V^{H}\right)$ and $\tilde{x}$ be an element on the unique closed orbit in $\pi^{-1}(x)$. Then its stabilizer $K$ is finite. Let $N$ be the slice representation at $\tilde{x}$, i.e., a $K$-stable complement of $T_{\tilde{x}} G \tilde{x} \subset T_{\tilde{x}} V=V$. The quotient map $N \rightarrow$ $N / / K$ for $K$ is equidimensional, so $\mathbf{k}[N]$ is a Cohen-Macaulay $\left(B^{\prime}:=\mathbf{k}[N]^{K}\right)$ module. $B^{\prime}$ is Gorenstein, since the finite group $K$ acts with determinant equal to one on $N$. Using Lemma 4.1 it follows that $\operatorname{Ext}_{B^{\prime}}^{i}\left(\mathbf{k}[N], B^{\prime}\right)=0$ if $i \neq 0$.

According to Luna's étale slice theorem (see [9]), there is an $f \in B$ with $f(x) \neq 0$ and a Cartesian diagram

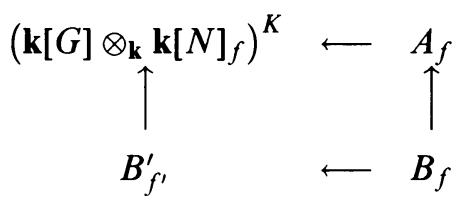

where the horizontal maps are faithfully flat; $K$ acts on $\mathbf{k}[G \times N] \simeq \mathbf{k}[G] \otimes_{\mathbf{t}}$ $\mathbf{k}[N]$ by $(k x)(g, n):=x\left(g k, k^{-1} n\right)$, with $k \in K, g \in G, n \in N_{f^{\prime}}$, and $x \in \mathbf{k}[G \times N]$. In particular,

$$
\left(\mathbf{k}[G] \otimes \mathbf{k}[N]_{f}\right)^{K} \simeq A_{f} \otimes_{B_{f}} B_{f}^{\prime} .
$$

So for every $G$-module $M$, we have for $i \neq 0$

$$
\begin{aligned}
\operatorname{Ext}_{B}^{i}(B(M), B)_{f} \otimes_{B_{f}} B_{f}^{\prime} & \simeq \operatorname{Ext}_{B_{f}^{\prime}}^{i}\left(\left(M \otimes_{\mathbf{k}} A_{f} \otimes_{B_{f}} B_{f}^{\prime}\right)^{G}, B_{f}^{\prime}\right) \\
& \simeq \operatorname{Ext}_{B_{f}^{\prime}}^{i}\left(\left(M \otimes_{\mathbf{k}} \mathbf{k}[G] \otimes_{\mathbf{k}} \otimes \mathbf{k}[N]_{f}\right)^{G \times H}, B_{f}^{\prime}\right) \\
& \simeq \operatorname{Ext}_{B_{f}^{\prime}}^{i}\left(\left(M \otimes_{\mathbf{k}} k[N]_{f}\right)^{H}, B_{f}^{\prime}\right) \simeq 0 .
\end{aligned}
$$

So $\operatorname{Ext}_{B}^{i}(A, B)_{f}=0$ and $x$ is not in the support of $\operatorname{Ext}_{B}^{i}(A, B)$.

The following lemma is very useful and is due to Brion [4]. 
Lemma 4.3. Suppose $\operatorname{dim} V / / G-e \geq 2$. Then $B(M) \simeq B\left(M^{*}\right) \simeq B(M)^{*}$ for all $G$-modules $M$.

Proof. We sketch the proof. Let $\phi$ be the composition

$$
B\left(M^{*}\right) \simeq \operatorname{Hom}_{A}(A \otimes M, A)^{G} \rightarrow \operatorname{Hom}_{B}(B(M), B)=B(M)^{*},
$$

where the second map is the restriction. As in the proof of Lemma 4.2 one checks that $\phi$ is an isomorphism outside $\pi\left(V^{H}\right)$. Since both $B\left(M^{*}\right)$ and $B(M)^{*}$ are reflexive and the codimension of $\pi\left(V^{H}\right)$ in $V / / G$ is $\operatorname{dim} V / / G-e \geq$ 2 , we conclude that $\phi$ is an isomorphism.

We apply the foregoing lemmas to give a direct proof of a fact observed before by Van den Bergh [1].

Proposition 4.1. Suppose $\operatorname{dim} V / / G-e \geq 2$ and let $M$ be any $G$-module. Then for $i \neq 0$ or $\tau-2$

$$
\operatorname{Ext}_{B}^{i}(B(M), B)=H_{B^{+}}^{d-i}(B(M))=0 .
$$

Proof. Let $P$ be the homogeneous prime ideal of $B$ corresponding to $\pi\left(V^{H}\right)$. The variety $X:=\pi^{-1} \pi\left(V^{H}\right)$ equals $G \cdot\left(\bigoplus_{j \geq 0} V_{j}\right)$, where $V_{j}$ is the $H$-weight space of weight $j$. Since $X$ is stabilized by the upper triangular matrices of $G$, the dimension of $X$ is $\tau+e+1$. Since $A$ is Cohen-Macaulay, we have

$$
\operatorname{depth}_{P}(A)=\operatorname{height}(P \cdot A)=\operatorname{dim} V-\operatorname{dim} X=\tau-1 .
$$

Suppose there is a $j>0$ such that $\operatorname{Ext}_{B}^{j}(B(M), B) \neq 0$, and take $j$ minimal. By dualizing a free resolution of $B(M)$ we get a sequence

$$
0 \rightarrow B(M)^{*} \rightarrow F_{0}^{*} \rightarrow \cdots \rightarrow F_{j}^{*} \rightarrow C \rightarrow 0,
$$

where all $F_{i}^{*}$ are free and $\operatorname{Ext}_{B}^{j}(B(M), B) \subset C$. Let $Q \supset P$ be an associated prime ideal of $\operatorname{Ext}_{B}^{j}(B(M), B)$; it is associated to $C$ as well, $\operatorname{depth}_{Q}(C)=0$, and by the depth lemma [5, Lemma 1.1] $\operatorname{depth}_{Q}\left(B(M)^{*}\right)=j+1$.

Since $B(M) \simeq B(M)^{*}$ by Lemma 4.3 , we have

$$
\begin{aligned}
j+1 & =\operatorname{depth}_{Q}\left(B(M)^{*}\right) \geq \operatorname{depth}_{P}\left(B(M)^{*}\right) \\
& =\operatorname{depth}_{P}(B(M)) \geq \operatorname{depth}_{P}(A)=\tau-1 .
\end{aligned}
$$

So $j \geq \tau-2$. By Lemma 4.1 we get $H_{B^{+}}^{d-j}(B(M))=0$ if $0<j<\tau-2$.

Since

$$
\operatorname{depth}_{B^{+}} A=\operatorname{height}\left(B^{+} A\right)=\operatorname{codim}_{V} \pi^{-1} \pi(0)=\operatorname{dim} V-\tau-1,
$$

we have $H_{B^{+}}^{d-j}(B(M))=0$ for $j>\tau-2$. We conclude that $\operatorname{Ext}_{B}^{j}(B(M), B)$ vanishes for $j>\tau-2$, by using Lemma 4.1 again. This proves the lemma. (This proof is inspired by the proof of [5, Theorem 3.8].)

\section{Classification of Cohen-Macaulay modules of covariants}

Let $C$ be the subalgebra of $B$ generated by a homogeneous system of parameters of degrees $\sigma_{1}, \ldots, \sigma_{d}$, and write $\sigma:=\operatorname{dim} V-\sum_{i=1}^{d} \sigma_{i}$. Since $B$ is a graded Gorenstein domain, by the theorem of Hochster and Roberts, $B$ is finitely generated and free as a $C$-module. 
Proposition 5.1. Suppose $\operatorname{dim} V / / G-e \geq 2$.

(i) Let $p \geq 0$, and if $e=m$ let $p$ be even. Then

$$
\mathscr{G}\left(\operatorname{Ext}_{B}^{\tau-2}\left(B\left(R_{p}\right), B\right) ; t\right)=(-1)^{\tau-2} \tilde{\mathscr{G}}_{p}=\frac{\psi_{p-s+2}(t) t^{-\tau}}{(1-t)^{e}} .
$$

(ii) Now suppose that $p \geq s-2$. Then $\operatorname{Ext}_{B}^{\tau-2}\left(B\left(R_{p}\right), B\right)$ is a CohenMacaulay $B$-module of dimension $e$.

(iii) There is a minimal free graded $C$-resolution of $B\left(R_{p}\right)$ of the form

$$
0 \rightarrow F_{\tau-2} \rightarrow F_{\tau-3} \rightarrow \cdots \rightarrow F_{1} \rightarrow F_{0} \rightarrow B\left(R_{p}\right) \rightarrow 0 .
$$

This resolution and its dual, shifted over $\sigma$ degrees, patch together to a minimal free graded C-resolution of $\operatorname{Ext}_{B}^{\tau-2}\left(B\left(R_{p}\right), B\right)$ :

$$
\begin{aligned}
0 & \rightarrow F_{\tau-2} \rightarrow \cdots \rightarrow F_{0} \rightarrow F_{0}^{*}[\sigma] \rightarrow F_{1}^{*}[\sigma] \rightarrow \cdots \rightarrow F_{\tau-2}^{*}[\sigma] \\
& \rightarrow \operatorname{Ext}_{B}^{\tau-2}\left(B\left(R_{p}\right), B\right) \rightarrow 0 .
\end{aligned}
$$

Proof. Let for the moment $F_{\bullet} \rightarrow B\left(R_{p}\right)$ be any free graded $C$-resolution, which is always of finite length. The homology group $H_{i}\left(F_{\bullet}^{*}\right)$ of the dual complex is $\operatorname{Ext}_{C}^{i}\left(B\left(R_{p}\right), C\right)$. Since by standard arguments $H_{C^{+}}^{d-i}\left(B\left(R_{p}\right)\right) \simeq H_{B^{+}}^{d-i}\left(B\left(R_{p}\right)\right)$, it follows by applying Lemma 4.1 twice that $\operatorname{Ext}_{C}^{i}\left(B\left(R_{p}\right), C\right)[\sigma]=$ $\operatorname{Ext}_{B}^{i}(B(M), B)$ as $B$-modules. By Proposition 4.1 there are at most two nonvanishing Ext-groups of which $\operatorname{Ext}_{B}^{0}\left(B\left(R_{p}\right), B\right) \simeq R_{p}$, by Lemma 4.3.

From

$$
\mathscr{G}\left(B\left(R_{p}\right), t\right)=\sum_{i}(-1)^{i} \mathscr{G}\left(F_{i}, t\right)
$$

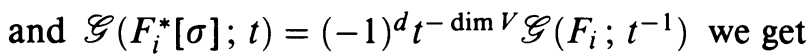

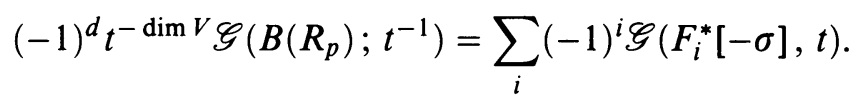

Since

$$
\sum_{i}(-1)^{i} \mathscr{G}\left(F_{i}^{*}[-\sigma], t\right)=\mathscr{G}\left(B\left(R_{p}\right)^{*}, t\right)+(-1)^{\tau-2} \mathscr{G}\left(\operatorname{Ext}_{B}^{\tau-2}\left(B\left(R_{p}\right), B\right), t\right),
$$

we get

$$
\begin{aligned}
& \mathscr{G}\left(\mathrm{Ext}_{B}^{\tau-2}\left(B\left(R_{p}\right), B\right) ; t\right)
\end{aligned}
$$

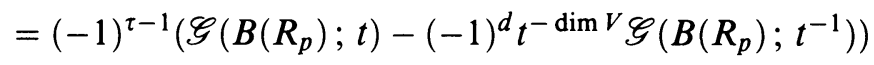

$$
\begin{aligned}
& =(-1)^{\tau-2} \widetilde{\mathscr{G}}_{p}=\frac{\psi_{p-s+2}(t) t^{-\tau}}{(1-t)^{e}},
\end{aligned}
$$

where we used Proposition 2.1. This proves (i).

By Lemma 3.1, $\operatorname{Ext}_{B}^{\tau-2}\left(B\left(R_{p}\right), B\right)$ does not vanish if and only if $p \geq s-2$ and, when $e=m, p$ is even . If it vanishes and $B\left(R_{p}\right)$ is not zero, then by Lemma 4.1 the depth of $B\left(R_{p}\right)$ is $d$; hence, $B\left(R_{p}\right)$ is free as a $C$-module.

From now on suppose $p \geq s-2$ and, when $e=m, p$ is even. Then the depth of $B\left(R_{p}\right)$ is $d-(\tau-2)$, so a minimal free graded $C$-resolution exists 
of length $\tau-2$. Taking its dual and shifting over $\sigma$ degrees, we get that

$$
0 \rightarrow B\left(R_{p}\right)^{*} \rightarrow F_{0}^{*}[\sigma] \rightarrow \cdots \rightarrow F_{\tau-2}^{*}[\sigma] \rightarrow \operatorname{Ext}_{B}^{\tau-2}\left(B\left(R_{p}\right), B\right) \rightarrow 0
$$

is exact. Since $B\left(R_{p}\right)^{*} \simeq B\left(R_{p}\right)$, the two free complexes glue together to a minimal free graded $C$-resolution of $\operatorname{Ext}_{B}^{\tau-2}\left(B\left(R_{p}\right), B\right)$, and so the depth of it is $2 \nu-d-1=e$. From the Hilbert series we see that its dimension is also $e$, so $\operatorname{Ext}_{B}^{\tau-2}\left(B\left(R_{p}\right), B\right)$ is a graded Cohen-Macaulay $B$-module.

5.1. The representations $V$ such that all its modules of covariants are CohenMacaulay are the same as those where the quotient map $\pi$ is equidimensional, so those where

$$
\operatorname{dim} V-\operatorname{dim} V / / G=\operatorname{dim} \pi^{-1} \pi(0)=\sum_{i=1}^{m}\left\lceil\frac{d_{i}}{2}\right\rceil+1=\tau+1 .
$$

So by some arithmetic, the result is that, up to trivial summands, $V$ is one of the representations contained in

$$
\left\{R_{1}, R_{1} \oplus R_{1}, R_{2}, R_{1} \oplus R_{2}, R_{2} \oplus R_{2}, R_{3}, R_{4}\right\} .
$$

In fact, in this case all modules of covariants are free (see [8]). They can also be characterized as the representations with $\operatorname{dim} V / / G-e<2$.

The full classification is given in the following theorem.

Theorem 5.1. (i) If $\operatorname{dim} V-d=\tau+1$ then all modules of covariants for $V$ are free.

(ii) Suppose $\operatorname{dim} V-d<\tau+1$. If $e=m$ then $B\left(R_{p}\right)=0$ when $p$ is odd. Let $p \in \mathbb{N}$ be even if $e=m$ and arbitrary if $e \neq m$. The following statements are equivalent:

(1) $p<s-2$;

(2) $B\left(R_{p}\right)$ is a Cohen-Macaulay B-module;

(3) the deviation $\tilde{\mathscr{G}}_{p}$ from Stanley's functional equation is zero;

(4) the degree of $\mathscr{G}\left(B\left(R_{p}\right)\right)$ as a rational function is smallar than or equal to $-\operatorname{dim} V$

(5) $H_{B^{+}}^{\tau+e-1}\left(B\left(R_{p}\right)\right)=0$; and

(6) $\operatorname{Ext}_{B}^{\tau-2}\left(B\left(R_{p}\right), B\right)=0$.

Proof. We discussed (i) just before the statement of the theorem. The equivalences follow from Proposition 5.1 using Propositions 3.1 and 4.1 and Lemma 4.1.

\section{REFERENCES}

1. M. Van den Bergh, A converse to Stanley's conjecture for $\mathrm{SL}_{2}$, preprint 89-38, Nov. 1989, Universitaire Instelling Antwerpen.

2. Cohen-Macaulayness of modules of covariants, Invent. Math 106 (1991), 389-409.

3. A. Broer, On the generating functions associated to a system of binary forms, Indag. Math. (N.S.) 1 (1990), 15-25.

4. M. Brion, Sur les modules des covariants, Ann. Sci. École. Norm. Sup. 26 (1993), 1-21.

5. E. G. Evans and P. Griffith, Syzygies, Cambridge Univ. Press, Cambridge, MA, 1985.

6. S. Goto and K. Watanabe, On graded rings. I, J. Math. Soc. Japan 30 (1978), 179-213. 
7. M. Hochster and J. L. Roberts, Rings of invariants of reductive groups acting on regular rings are Cohen-Macaulay, Adv. in Math. 13 (1974), 115-175.

8. G. W. Schwarz, Representations of simple Lie groups with a free module of covariants, Invent. Math. 50 (1978), 1-12.

9. P. Slodowy, Der Scheibensatz für algebraische Transformationsgruppen, Algebraische Transformationsgruppen und Invariantentheorie. Algebraic Transformation Groups and Invariant Theory (H. Kraft, P. Slodowy, and T. A. Springer, eds.), DMV Seminar, Band 13, Birkhäuser Verlag, Basel, 1989.

10. R. P. Stanley, Combinatorics and invariant theory, Proc. Sympos. Pure Math., vol. 34, Amer. Math. Soc., Providence, RI, 1979, pp. 345-355.

Department of Mathematics, University of British Columbia, 1984 Mathematics Road, Vancouver, British Columbia, Canada V6T 1 Z2

E-mail address: broer@unixg.ubc.ca 